\title{
Editorial
}

\section{Special Issue on Selected Papers from IVAPP 2018}

\author{
Alexandru Telea ${ }^{1, *}$ and Andreas Kerren ${ }^{2, *}$ \\ 1 Bernoulli Institute for Mathematics, Computer Science and Artificial Intelligence, University of Groningen, \\ P.O. Box 72, 9700 AB Groningen, The Netherlands \\ 2 Department of Computer Science and Media Technology, Linnaeus University, 35195 Växjö, Sweden \\ * Correspondence: a.c.telea@rug.nl (A.T.); andreas.kerren@lnu.se (A.K.)
}

Received: 12 July 2018; Accepted: 12 July 2018; Published: 13 July 2018

Recent developments at the crossroads of data science, data mining, machine learning, and graphics and imaging sciences have further established information visualization and visual analytics as central disciplines that deliver methods, techniques, and tools for making sense of and extracting actionable insights and results from large amounts of complex, multidimensional, hybrid, and time-dependent data. The International Conference on Information Visualization Theory and Applications (IVAPP), which reached its ninth edition in 2018, and was held on 27-29 January in Funchal, Madeira, Portugal, has become an established international meeting point for researchers, engineers and practitioners in all subfields of information visualization. The conference program featured twelve full papers describing advanced prototypes, systems, tools, and techniques related to the theory and application of information visualization in fields.

This Special Issue aims to offer a platform for the best papers presented at IVAPP 2018 to expand upon their results. Submissions were open to a subset of the full IVAPP 2018 papers, selected by the Special Issue editors based on the conference reviews, the presentation, and the expected impact on the research community. Each Special Issue submission was mandated to contain at least $50 \%$ new material in the form of technical extensions, more in-depth evaluations, or additional use cases. The submissions underwent a peer-review process, in which at least two members of the IVAPP 2018 program committee acted as reviewers. Where and when needed, additional external reviewers were invited to guarantee the high quality of the reviewing process.

The Special Issue received four submissions, all of which were accepted after several reviewing cycles. The accepted papers effectively illustrate current challenges, points of interest, and developments in information visualization, most notably regarding the summarization and exploration of large datasets or data collections.

Multidimensional or multi-field data collections are a particular challenge for practitioners and researchers interested in extracting insights and exploring these efficiently and effectively. The first paper entitled "A Top-Down Interactive Visual Analysis Approach for Physical Simulation Ensembles at Different Aggregation Levels" [1] addresses this challenge for the use case of exploring a large collection of multi-field, multi-run, time-dependent datasets obtained by executing several simulation runs for different parameter values. For this, the authors propose a set of different visual encodings (histograms, density-based time series plots, and similarity-based plots) that allow the exploration of the large data corpus at hand at multiple levels of detail. While demonstrated on use cases from climate research and astrophysics simulations, the proposed approach is also generically applicable to data coming from other application domains.

A different way to cope with large multidimensional data is proposed by the second paper entitled “Upsampling for Improved Multidimensional Attribute Space Clustering of Multifield Data" [2]. Here, the authors propose a range of techniques to improve the reliability of clustering algorithms typically used to create summarized representations of multidimensional datasets. Recognizing that clustering is sensitive to the count and distribution of available data samples, they propose 
an upsampling technique that efficiently and effectively scans the high-dimensional data space and adds extra sample points by using various interpolation techniques that aim to preserve underlying data characteristics such as smoothness and/or sharp boundaries. They demonstrate that their proposal can effectively improve the reliability and decrease the sensitivity to parameter settings for traditional histogram-based clustering methods.

The third paper entitled "An Evolutionary Algorithm for an Optimization Model of Edge Bundling" [3] focuses on relational, rather than multidimensional, data. For coping with the (summarized) presentation and visual analysis of large datasets of this type, edge bundling has already become the established method. However, most edge bundling methods in existence are based on heuristics that often do not have an explicit optimization criterion in focus. The paper addresses this issue by casting edge bundling as a set of three optimization problems, and next proposes an evolutionary algorithm to solve these. Besides the novel formal interpretation of edge bundling in optimization terms, the paper also shows novel practical results, such as ways to minimize the number of produced bundles from a given input dataset.

The fourth and final paper entitled "More Compact Orthogonal Drawings by Allowing Additional Bends" [4] stays in the domain of relational data visualization, but addresses a different problem, namely the computation of high-quality orthogonal drawings of graphs. As a quality criterion, the compactness, or planar area needed to draw a given graph, is considered. The paper proposes the relaxation of known layout heuristics in orthogonal graph drawing by allowing the introduction of a limited number of additional edge bends. By doing this, the authors show that the overall compactness criterion (measured as a function of the total edge length and the total drawing area needed) can be measurably improved, leading to more scalable drawing algorithms. The paper also proposes a novel algorithm for solving the orthogonal graph drawing problem based on a network flow model.

Besides the common focus of all Special Issue papers on visualizing increasingly larger and/or more complex data, all papers also allocate considerable space for presenting both quantitative and qualitative evaluations of their proposed methods and techniques, in terms of comparisons with competing methods, evaluations on multiple datasets and use cases, involvement of domain experts in the evaluations, and usage of quantitative quality metrics in the evaluation. We see this as a welcome development in information visualization, which will lead to both higher quality but also more replicable research results.

The guest editors Alexandru Telea and Andreas Kerren would like to thank the authors for their contributions and the reviewers for their effort in providing valuable comments during the revision process.

Conflicts of Interest: The authors declare no conflict of interest.

\section{References}

1. Fofonov, A.; Linsen, L. A Top-Down Interactive Visual Analysis Approach for Physical Simulation Ensembles at Different Aggregation Levels. Information 2018, 9, 163. [CrossRef]

2. Molchanov, V.; Linsen, L. Upsampling for Improved Multidimensional Attribute Space Clustering of Multifield Data. Information 2018, 9, 156. [CrossRef]

3. Ferreira, J.D.M.; Do Nascimento, H.A.; Foulds, L.R. An Evolutionary Algorithm for an Optimization Model of Edge Bundling. Information 2018, 9, 154. [CrossRef]

4. Jünger, M.; Mutzel, P.; Spisla, C. More Compact Orthogonal Drawings by Allowing Additional Bends. Information 2018, 9, 153. [CrossRef]

(C) 2018 by the authors. Licensee MDPI, Basel, Switzerland. This article is an open access article distributed under the terms and conditions of the Creative Commons Attribution (CC BY) license (http://creativecommons.org/licenses/by/4.0/). 